\title{
Gerbu adjuvant modulates the immune response and thus the course of infection in C56BL/6 mice immunised with Echinococcus multilocularis rec14-3-3 protein
}

\author{
Maxi Margos • Bruno Gottstein
}

Received: 5 April 2010 /Accepted: 29 April 2010 /Published online: 19 May 2010

(C) Springer-Verlag 2010

\begin{abstract}
Vaccination with Echinococcus multilocularis 14-3-3 protein can protect mice against primary E. multilocularis infection. The present study investigated the efficacy and efficiency of the adjuvant muramyl dipeptide Gerbu, alone or together with recombinant 14-3-3 protein, to modulate the course of secondary E. multilocularis infection in C56BL/6 mice. The application of Gerbu alone already resulted in a parasite weight reduction when compared with infected control mice, while rec14-3-3 did not add to this effect. Immunological parameters were concurrently assessed with a mixed cell reaction including bone marrow-derived dendritic cells (BMDCs) together with lymph node cells from mice with or without immunisation and/or infection. While mice having received Gerbu adjuvant were found to highly proliferate in response to co-cultivation with 14-3-3-stimulated bone marrow dendritic cells, a sensitisation of BMDCs with vesicle fluid (VF) antigen lead to a striking decrease of the lymphoproliferative response in comparison to that of control mice, raising the hypothesis that immunosuppressive components may be part of this VF-antigen. Anti-14-3-3 antibody production was only found in those mice that had been previously 14-3-3-immunised, whereas all other only-infected mice failed to produce such antibodies. Conclusively, Gerbu adjuvant appears to directly generate a non-specific immune response that contributes to the control of the metacestode growth, putatively in association with a BMDC activity suppressed by components of the VF-antigen.
\end{abstract}

M. Margos $\cdot$ B. Gottstein $(\triangle)$

Institute of Parasitology, University of Berne,

Länggass-Strasse 122,

CH-3012 Bern, Switzerland

e-mail: bruno.gottstein@ipa.unibe.ch

\section{Introduction}

Secondary experimental infection with Echinococcus multilocularis in mice goes along with an intense host-parasite interplay orchestrated predominantly by the parasite in view to provide survival (Bresson-Hadni et al. 1990; Emery et al. 1996; Vuitton et al. 1989). The proliferating metacestode of E. multilocularis consists of an inner, germinal layer, representing the living parasite, sheathed by an outer acellular laminated layer providing protection against effector mechanisms of the host (Gottstein and Hemphill 1997). Protection may also depend on immunemodulatory effects of parasite metabolites (Gottstein and Hemphill 1997; Meijri et al. 2010). How this modulation or evasion is achieved is not yet fully elucidated, either for Echinococcus granulosus (Diaz et al. 1997) or for E. multilocularis (Vuitton and Gottstein 2010). Known parasite molecules of respective relevance include, e.g. a carbohydrate antigen, Em2(G11), which acts as an T cellindependent antigen and therefore inhibits maturation of antibody avidity (Dai et al. 2001). Walker et al. (2004) found the Em492-antigen, a carbohydrate molecule with biological activity, supposed to exhibit immunosupressive properties at the host-parasite interface. Metacestodic antigens [such as vesicle fluid (VF) from $E$. multilocularis] contain substances responsible for the inactivation of eotaxin by proteolytic activity in vitro. This inactivation may be relevant for the parasites survival, as eotaxin works as a main activator and chemo-attractant for eosinophils, and the impairment of eosinophils to infiltrate the site of parasite residence seems to be an important parameter in avoiding the host defense (Mejri and Gottstein 2009). Similar other studies revealed that other secreted metacestode products influence the outcome of an infection in favour of the parasite as well 
(Allen and MacDonald 1998; Lightowlers and Rickard 1988; Pastrana et al. 1998).

In the case of primary (peroral egg) E. multilocularis infection, first trials had revealed that rec14-3-3 is able to induce protection in mice against the oncosphere, but not against a mature larval metacestode (Siles-Lucas et al. 2003). The protective immune response, yielded upon immunisation with Saponin as adjuvant, was not polarised, e.g. specific antibodies as well as cytokines accounting for both Th1 and Th2 orientation were found (Siles-Lucas et al. 2003). In the present study, we addressed the question, how a more potent adjuvant (Gerbu adjuvant) interacts with the host immune system, in relation to different types of $E$. multilocularis antigens.

\section{Materials and methods}

Mice

Female C56/BL6 mice were purchased from Charles River, Germany. Average age of animals was 8-10 weeks when mice received the first immunisation. All mice were handled under standard animal laboratory conditions according to Swiss regulations for animal experimentation.

\section{Parasite antigens}

E. multilocularis VF-antigen was obtained by gentle disruption of in vitro maintained vesicles, following centrifugation and recovery of supernatant (Ingold et al. 1999). The protein concentration of the VF batch used in the present study was $0.94 \mathrm{mg} / \mathrm{ml}$. E. multilocularis rec14-3-3 antigen was obtained and purified as described before (Dai and Gottstein 1999; Siles-Lucas et al. 1998), the batch concentration was $0.17 \mathrm{mg}$ protein per millilitre.

Immunisation of C57BL/6 mice and infection with $E$. multilocularis metacestodes

For immunisation $10 \mu \mathrm{g}$ rec14-3-3 were mixed 1:1 with Gerbu adjuvant (GMDP; Biotechnik GmbH, Gaiberg, Germany) to yield a final inoculation volume of $100 \mu 1$ per injection. Each group of mice $(n=5)$ were subcutaneously injected at the shoulder site. A control group received a mixture of ovalbumin $(10 \mu \mathrm{g}$ per injection, Dr. Grogg AG) and Gerbu, and another group Gerbu mixed with an equal volume of phosphate-buffered saline (PBS). Non-treated control mice received PBS only. Booster injection ensued 2 weeks later. Another 14 days later, appropriate groups of mice were infected intraperitoneally with $E$. multilocularis clone KF5as described before (Dai and Gottstein 1999); the other groups remained non-infected. Three months post-infection mice were killed by $\mathrm{CO} 2$-euthanasia.

Parasite weighing

The parasite tissue (metacestode mass) was removed carefully from the peritoneal cavity, freed of any attached hosts' tissue and weighted.

\section{Cultivation of BMDCs}

Naive C56BL/6 animals were killed and bone marrow progenitors were isolated and cultured as described elsewhere (Lutz et al. 1999). Culturing took place in Petri dishes (Greiner, 145/20) with feeding of the cells every 3 days with $5 \mathrm{ml}$ RPMI 1640 medium (GIBCO Invitrogen) and $200 \mathrm{U}$ rh GM-CSF (Immunotools, Friesoythe, Germany). On day 9, non-adherent cells were harvested and, after counting, adjusted to a concentration of $1 \times 10^{6}$ cells per millilitre. Cells were plated in 12-well plates and supplemented with medium without GM-CSF for further experiments. Cell preparation contained $75 \%$ to $80 \% \mathrm{CD}_{11 \mathrm{c}^{+}}$bone marrow-derived dendritic cells (BMDCs) as determined with fluorometric analysis.

Stimulation of immature DCs was carried out as described below using the following antigens:

- VF $(2 \mu \mathrm{g} / \mathrm{ml})$

- Rec14-3-3 antigen $(1 \mu \mathrm{g} / \mathrm{ml})$

- $100 \mathrm{ng} / \mathrm{ml}$ lipopolysaccharide (LPS) as a positive control

- Appropriate volume of medium alone as a negative control

Immature dendritic cells (DCs) were exposed to stimulation with the antigens as described above and maintained for 16-18 h. Cells were subsequently collected and washed with warm supplemented medium.

\section{Cell proliferation assay}

Lymph node (LN) cells (renal, inguinal, lumbar, and brachial) from control and treated (immunised with rec14-3-3, Gerbu, ovabulmin, and PBS and later with or without E. multilocularis metacestode infection) C56/ BL6 mice were isolated. LN cells $\left(1 \times 10^{6} / \mathrm{ml}\right)$ were cocultivated with BMDCs $\left(1 \times 10^{5} / \mathrm{ml}\right)$ from naive or $E$. multilocularis-infected mice. These BMDCs had been cultured before in medium alone or in medium plus stimulation with LPS or the three parasite antigens for $18 \mathrm{~h}$ and washed thereafter. After 5 days of co-cultivation, cells were pulsed with $1 \mu \mathrm{Ci} /$ well ${ }^{3} \mathrm{H}$ thymidine. After $18 \mathrm{~h}$, the cells were harvested onto glass fibre filters and activity measured in a beta counter (PerkinElmer) upon thymidine incorporation. 


\section{IgG ELISA}

Sera of mice were obtained on the day before infection and on the day of being killed. Sera of day 0 (before vaccination) served as negative controls, and positive control sera were used from animals of a previous experiment (Siles-Lucas et al. 1998). The presence of anti-E. multilocularis 14-3-3 and anti-Em2(G11) [outgroup control antigen] IgG antibodies was assessed by enzyme-linked immunosorbent assay (ELISA) as previously described (Dai and Gottstein 1999; Siles-Lucas et al. 1998). Alkaline phosphatase-conjugated goat anti-mouse IgG (Promega, Duebendorf, Switzerland), anti-IgG1, or anti-IgG2a (both from Southern Biotechnology Associates, Birmingham, USA) was used as second antibody (conjugate). Absorbance was measured at $405 \mathrm{~nm}\left(\mathrm{~A}_{405}\right.$, reference filter $630 \mathrm{~nm}$ ) using a Dynatech MR 7000 ELISA reader and the corresponding Dynatech Biocalc software (Dynatech, Embrach, Switzerland). The threshold value arbitrarily discriminating between "positive" and "negative" (cutoff) was defined by adding three standard deviations to the mean $\mathrm{A}_{405}$ value of the sera from non-infected and nontreated control mice.

\section{Results}

Parasite weight

The average parasitic mass recovered from PBS-control and subsequently, from E. multilocularis-infected mice was 1,930 mg (Fig. 1). Rec14-3-3-immunised-and-infected mice disclosed a significantly lower parasite weight with an average of $368 \mathrm{mg}$, as well as mice "control-immunised" with Gerbu adjuvant only and subsequently infected with $E$. multilocularis (average 442 mg; Fig. 1).

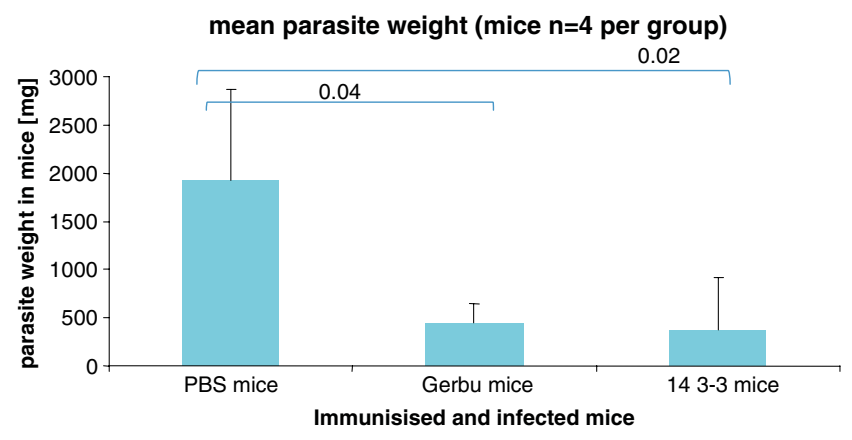

Fig. 1 Parasite weight recovered from C56BL/6 mice experimentally infected with $E$. multilocularis. Mice were either mock-immunised with PBS only or received Gerbu adjuvant supported immunisation with or without rec14-3-3-antigen. Horizontally labelled bars indicate $p$ values for statistical significances
Lymphoproliferative responses

The background proliferation activity of LN cells from 14-3-3 immunised mice was slightly elevated after the contact with immature BMDCs (Fig. 2a), indicating a potential contamination of the recombinant antigen with bacterial lipopolysaccharides. Otherwise, all control reactions with immature BMDCs yielded expected low background reactivities. LPS-maturated BMDCs originating from all mouse groups resulted in an elevated LN cell proliferation when compared with the corresponding activities of

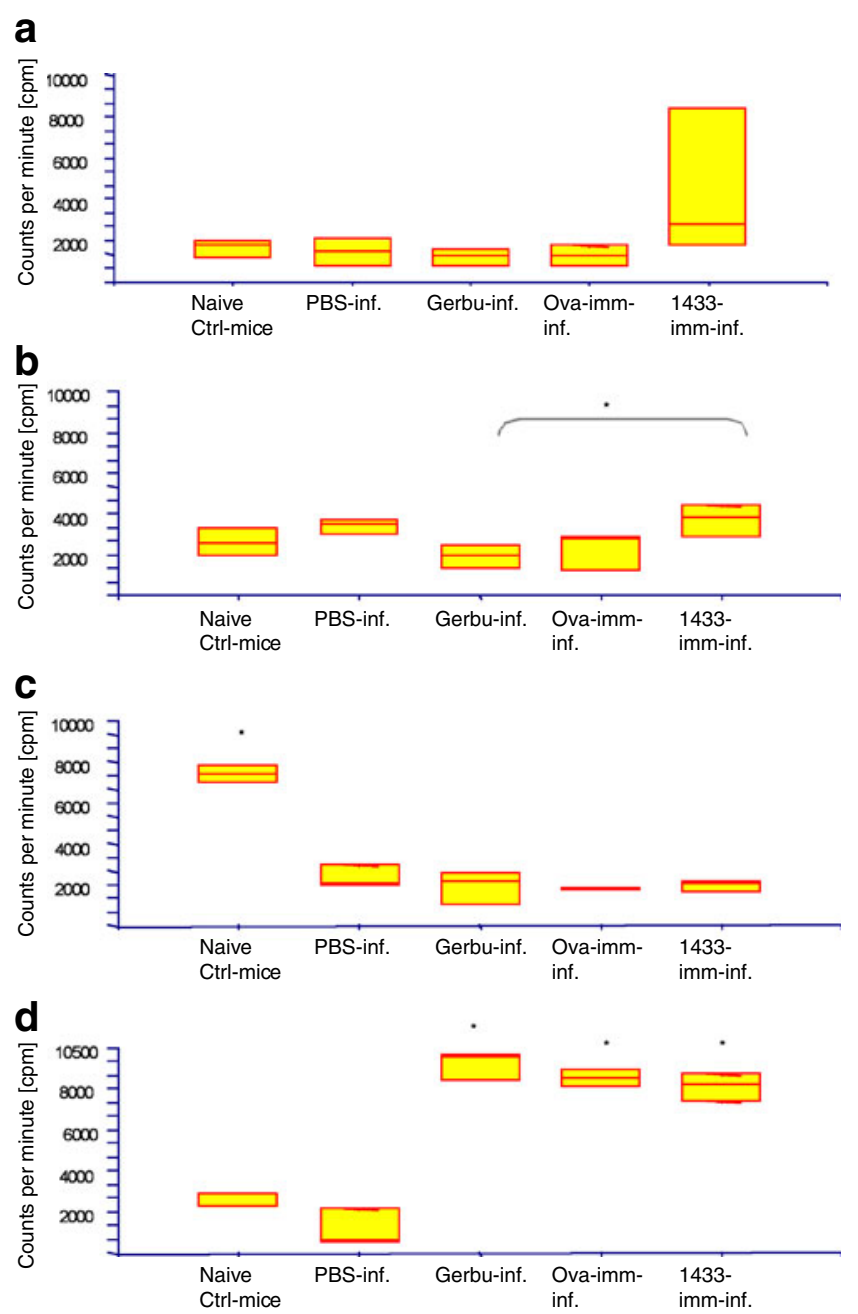

Fig. 2 Mixed BMDCs-lymphoproliferative assays. BMDCs were initially either kept with medium alone (immature DCs, iDCs) (a), or challenged for 16-18 h with LPS (b), VF (c), or rec14-3-3 (d), all iDCs were subsequently co-cultured with $\mathrm{LN}$ cells isolated from the following mouse groups: non-immunised/non-infected mice (naive Ctrl mice); E. multilocularis-infected mice: initially treated with PBS as a control (PBS-inf.), Gerbu-alone (Gerbu-inf.), ovabulmin plus Gerbu- (Ova-imm-inf.), or 14-3-3-plus Gerbu (14-3-3-imm-inf). Mice were killed 3 months after infection, control mice also on the same day. Incorporation of tritium-labelled thymidine was expressed as counts per minute (cpm). Statistical significances are labelled with an * and were at $p<0.05$ 
immature DCs (Fig. 2b). The highest reaction to LPSmaturated DCs was again seen in the 14-3-3 LN cells. VFpresentation by BMDCs resulted in high proliferative activity of only LN cells from naive mice (significantly higher to all other groups), whereas all other mouse groups exhibited only moderate to low proliferative responses (Fig. 2c). BMDCs stimulated with 14-3-3 resulted in a high proliferative response of LN cells from all three immunised-and-infected mice (all co-exposed to the Gerbu adjuvant). Conversely, LN cells from noninfected control animals and PBS-control-and-infected mice demonstrated an only very moderate response.

\section{IgG ELISA}

Serological analyses were carried out after 14-3-3-immunisation and later after immunisation plus 3 months of infection. ELISA was used to determine anti-14-3-3 IgG antibodies and antibodies to an outgroup control antigen [Em2(G11)]. Mice immunised with rec14-3-3 without or not yet infected showed no antibody production to control Em2(G11)-antigen, while 14-3-3-immunised mice, as expected, presented a marked anti-14-3-3-specific antibody reactivity (Fig. 3b). All infected mice revealed a marked anti-Em2(G11) antibody response, while anti-rec14-3-3 antibodies were only detectable in mice previously immunised with 14-3-3 (Fig. 3a), thus demonstrating that

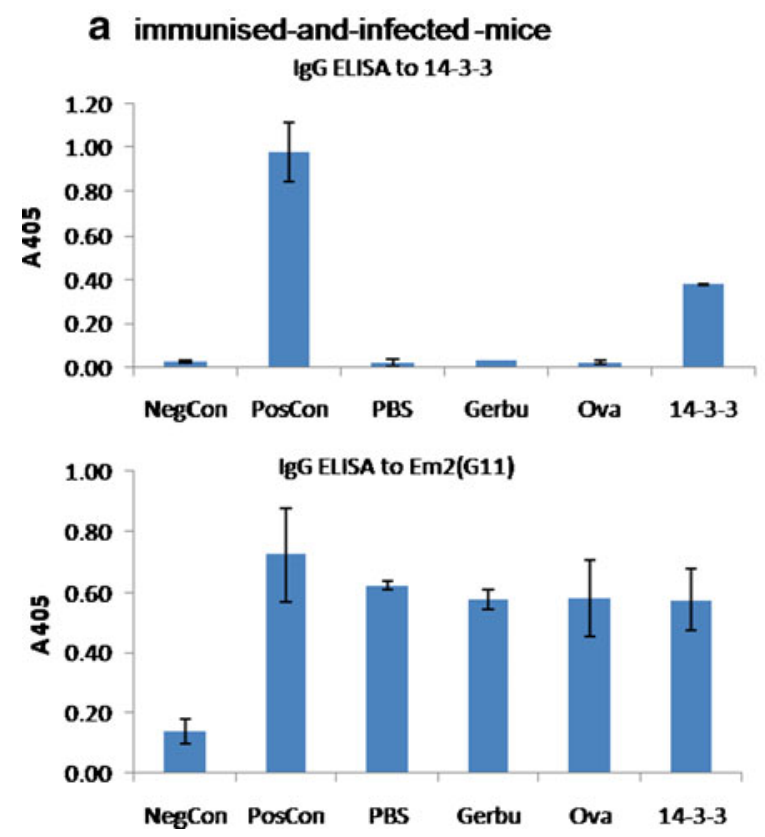

Fig. 3 ELISA-based analyses of serological antibody reactivities against rec14-3-3-antigen or against an outgroup control antigen Em2 (G11). a immunised-and-infected mice, b mice only immunised. Positive and negative control sera are labelled as NegCon and PosCon, respectively. $P B S$-group: mice receiving a mock- intraperitoneal E. multilocularis infection alone does not yield an anti-14-3-3-antibody production.

\section{Discussion}

Former studies (Reuben and Tanner 1983; Siles-Lucas et al. 2003) provided evidence of distinct differences between the initial anti-oncospheral immune response and the subsequent immune response directed against an established, maturated and fully active metacestode of E. multilocularis. Siles-Lucas et al. (2003) also showed that vaccination with rec14-3-3 yielded a good protection against primary E. multilocularis infection (peroral egg infection), while this immunity was ineffective against secondary infection (intraperitoneal inoculation of mature metacestodes). The interplay between a mature metacestode (chronic stage of infection/disease) and its murine host was intensively studied by multiple research groups (reviewed, e.g. by Gottstein and Hemphill 2008; Mejri and Gottstein 2009; Vuitton 2003) and had provided insight into the mode the parasite is involved in the modulation of the host immunity. The question we now presently addressed was if 14-3-3, though not inducing protection in mice secondarily infected with the metacestode, influences nevertheless the immune response thus as to yield a different immune recognition pattern when compared with nonimmunised animals. During our first experiments, we

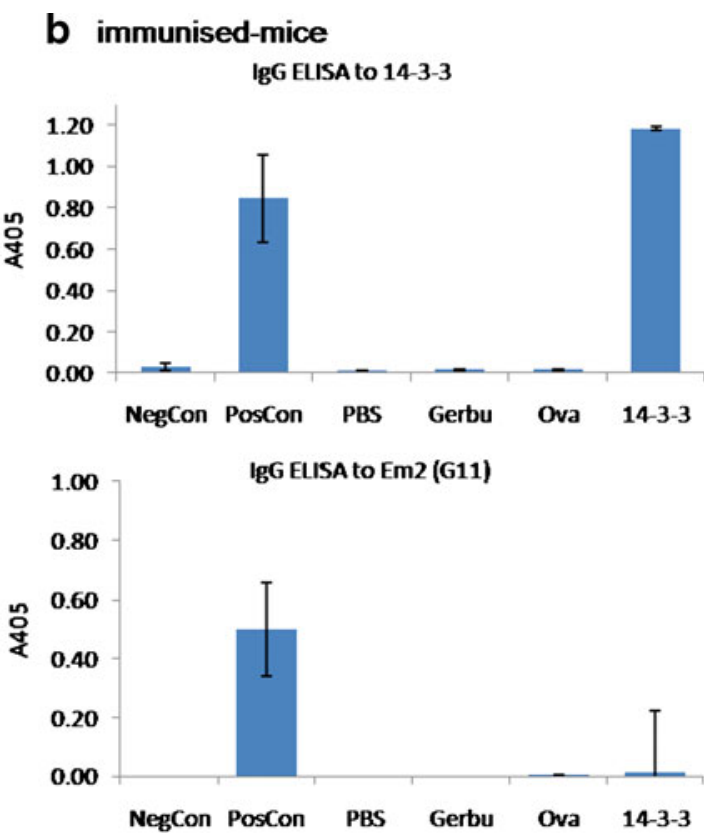

immunisation with PBS alone; Gerbu-group: mice receiving Gerbu adjuvant without antigen; Ova-group: mice immunised with Gerbu and ovalbumin; 14-3-3-group: mice immunised with Gerbu and rec143-3-antigen 
replaced the formerly used adjuvant saponin (Siles-Lucas et al. 2003) with the synthetic immunoadjuvant Gerbu. Gerbu is a muramyl dipeptide (MDP) representing the smallest subunit of bacterial cell walls still active as an adjuvant (Adam and Lederer 1984). Gerbu originates from Grampositive cell walls and displays a good alternative to common adjuvants, as it can be injected directly and safely in vivo. Conversely to saponin, immunisation with Gerbu and subsequent secondary infection yielded in a reduction of metacestode growth when being compared with nonimmunised alveolar echinococcosis (AE)-infected mice. This effect was independent of an additional use of antigen such as rec14-3-3 that we employed in the present study. At the early beginning of immunological $E$. multilocularis research, Reuben et al. (1978) had already revealed that the use of Bacillus Calmette-Guérin (BCG) (originating from an attenuated Mycobacterium bovis strain and inducing variable protection against tuberculosis and cancer) and also its cell wall skeleton (CWS) were inducing protection in cotton rats against the metacestode stage of infection. The use of lipoprotein from Mycobacterium tuberculosis is known to induce an effective Th1 cell-mediated immune response by TLR2 activation (Thoma-Uszynski et al. 2001), and so protection may be associated with the induction of a proper Th1 response. The former findings upon use of BCG or BCG-CWS had revealed a dose-dependent degree of protection; a low dose was not effective while a too-high dose formed tubercular granulomas in the host. So far, we have not addressed a dose-dependence of the Gerbu-effect in experimental murine AE. So far, most groups agreed that secondary murine $\mathrm{AE}$ includes a mixed $\mathrm{Th} 1 / \mathrm{Th} 2$ response, so the working hypothesis discussed by several authors was that partial protection (=reduced metacestode proliferation) may be associated with a rather Th1-orientation of immune response. A Th2-orientation may be in favour of metacestode proliferation (Amiot et al. 1999; Emery et al. 1996; Godot et al. 2003; Jenne et al. 1998; Liance et al. 1998).

The results showing reduced parasite masses in both Gerbu and Gerbu-rec14-3-3 immunised mice are in congruence with those of the lymphocyte proliferation assays. We found that stimulation of BMDCs cultivated with rec14-3-3 protein of E. multilocularis, and the following combination of these cells with LN cells from Gerbutreated mice resulted in antigen recognition and so to a significant LN cell proliferation. Conversely, non-Gerbutreated groups showed no reactivity to 14-3-3 stimulation. This raises the question whether this adjuvant does somehow interfere with the parasite or the mode the parasite exerts immune modulation of the host. Investigation of the humoral immune response also provided data as expected. First, sera obtained after rec14-3-3-immunisation showed the presence and thus proved synthesis of antirec14-3-3 IgGs, whereas no antibodies against the outgroup control antigen $\operatorname{Em} 2(\mathrm{G} 11)$ were yet detectable. Anti-Em2 (G11) IgG levels were positive in the sera of all immunisedand-infected or only-infected mice, while naive (non-infected control) mice produced no anti-Em2(G11) antibodies, as expected. Immuno-stimulating activity of vaccine adjuvants, such as muramyl dipeptides, may not only orchestrate the mode of antigen delivery to immunological sites, but may also influence the induction of secondary signals like B-7 family receptors and CD40 on APCs, directly or indirectly (Schijns 2000). This will be the focus of forthcoming studies we will carry out on the expression of co-stimulatory molecules compared between BMDCs from naive versus infected mice after different stimulations. MDP and derivates are strong inducers of a variety of cytokines such as IL-1, IL-6, TNF $\alpha$, and IFN- $\gamma$ in mice and humans and are therefore activating different cell types including Th1, Th2, or Th17 (de Jong et al. 2010). Another point of investigation we plan to undertake is to analyse the cytokine production pattern of cultured BMDCs stimulated in vitro with Gerbu adjuvant. The recognition of rec14-3-3 by LN cells from Gerbu-treated mice may be induced by unblocking of certain receptors. An interesting presumption by Rock et al. (1998) is that mycobacterial adjuvants do serve as ligands for receptors on immune competent cells, inducing marked cellular responses. At an early beginning of $\mathrm{AE}$ infection, the parasite is assumed to already start its modulation of the hosts' immune response such as to allow its subsequent survival. The accompanying innate immune system, largely non-specific and constitutively expressing pathogen recognition receptors for the recognition of pathogen-associated microbial patterns (PAMPs), may also be involved in the subsequent modulation of the specific immune response (Gerard et al. 1998). In vivo, E. multilocularis is expressing the carboxylated protein 14-3-3 in its germinal layer. In our present study, however, this antigen was presented by respectively in vitro-stimulated BMDCs such as to induce a subsequent lymphocyte proliferation. BCG is known to activate multiple Toll-like receptors (TLR; Azuma and Seya 2001); Gerbu also may function as a ligand for TLRs, interfering in the parasites reaction by uncovering different TLRs, so putatively miscasting the parasites modulation efficacy and thus proliferation (growth) efficiency. In a similar context, several authors (Azuma and Seya 2001) favoured a hypothesis in which TLR receptors may induce cellular responses supported by similar signaling events. The lack of TLR ligation/activation was supposed to initiate a Th2-oriented humoral immune response, which in E. multilocularis-infected man and mice may-at least partiallyaccount for the AE-specific (mixed Th1/Th2) immune orientation. Several MDP derivates also revealed the ability of re-stimulating immuno-suppressed mice, rats, and guinea pigs (Osada et al. 1982). A variety of TLRs were found to be expressed on DCs (Rock et al. 1998), allowing DCs to 
recognise PAMPs, like LPS. Furthermore, a large panel of TLRs is expressed on DC surfaces, like TLR-2, which is generally thought to be the receptor for Gram-positive bacteria (Knapp et al. 2002). In view of future experiments, we may address the different steps that account for Gerbu or other adjuvants to influence the activation status of DCs from naïve or AE-infected mice. Schijns (2000) had already proposed that, beside several signals which are important for DC activation, along with antigen presentation and the delivery of co-stimulatory molecules and cytokines, adjuvants may act as an induction factor, too. Another aspect to study as a result from our findings is that of putative therapeutic effects of amphotericin B and other antibiotics, which showed synergistic effects in combination with MDP derivates (Rock et al. 1998). As treatment with amphotericin $\mathrm{B}$ already showed a reduction in vesicle proliferation in vitro (Reuter et al. 2003), a promising approach could arise from enhancing the treatment efficacy by co-stimulation with an appropriate adjuvant.

Acknowledgements This work was supported by the Swiss National Science Foundation (Grant no. 31-111780/1). We kindly acknowledge the invaluable intellectual and experimental support provided by many collaborative colleagues, including Andrew Hemphill, Norbert Müller, and many others.

\section{References}

Adam A, Lederer E (1984) Muramyl peptides: immunomodulators, sleep factors, and vitamins. Med Res Rev 4:111-152

Allen JE, MacDonald AS (1998) Profound suppression of cellular proliferation mediated by the secretions of nematodes. Parasite Immunol 20:241-247

Amiot F, Vuong P, Defontaines M, Pater C, Dautry F, Liance M (1999) Secondary alveolar echinococcosis in lymphotoxin-alpha and tumour necrosis factor-alpha deficient mice: exacerbation of Echinococcus multilocularis larval growth is associated with cellular changes in the periparasitic granuloma. Parasite Immunol $21: 475-483$

Azuma I, Seya T (2001) Development of immunoadjuvants for immunotherapy of cancer. Int Immunopharmacol 1:1249-1259

Bresson-Hadni S, Liance M, Meyer JP, Houin R, Bresson JL, Vuitton DA (1990) Cellular immunity in experimental Echinococcus multilocularis infection. II. Sequential and comparative phenotypic study of the periparasitic mononuclear cells in resistant and sensitive mice. Clin Exp Immunol 82:378-383

Dai WJ, Gottstein B (1999) Nitric oxide-mediated immunosuppression following murine Echinococcus multilocularis infection. Immunology 97:107-116

Dai WJ, Hemphill A, Waldvogel A, Ingold K, Deplazes P, Mossmann H, Gottstein B (2001) Major carbohydrate antigen of Echinococcus multilocularis induces an immunoglobulin $\mathrm{G}$ response independent of alphabeta+ CD4+ T cells. Infect Immun 69:6074-6083

de Jong E, Suddason T, Lord GM (2010) Translational mini-review series on Th17 cells: development of mouse and human T helper 17 cells. Clin Exp Immunol 159:148-158

Diaz A, Ferreira A, Sim RB (1997) Complement evasion by Echinococcus granulosus: sequestration of host factor $\mathrm{H}$ in the hydatid cyst wall. J Immunol 158:3779-3786
Emery I, Liance M, Deriaud E, Vuitton DA, Houin R, Leclerc C (1996) Characterization of T-cell immune responses of Echinococcus multilocularis-infected C57BL/6J mice. Parasite Immunol 18:463-472

Gerard CJ, Olsson K, Ramanathan R, Reading C, Hanania EG (1998) Improved quantitation of minimal residual disease in multiple myeloma using real-time polymerase chain reaction and plasmidDNA complementarity determining region III standards. Cancer Res 58:3957-3964

Godot V, Harraga S, Podoprigora G, Liance M, Bardonnet K, Vuitton DA (2003) IFN alpha-2a protects mice against a helminth infection of the liver and modulates immune responses. Gastroenterology 124:1441-1450

Gottstein B, Hemphill A (1997) Immunopathology of echinococcosis. Chem Immunol 66:177-208

Gottstein B, Hemphill A (2008) Echinococcus multilocularis: the parasite-host interplay. Exp Parasitol 119:447-452

Ingold K, Bigler P, Thormann W, Cavaliero T, Gottstein B, Hemphill A (1999) Efficacies of albendazole sulfoxide and albendazole sulfone against in vitro-cultivated Echinococcus multilocularis metacestodes. Antimicrob Agents Chemother 43:1052-1061

Jenne L, Kilwinski J, Radloff P, Flick W, Kern P (1998) Clinical efficacy of and immunologic alterations caused by interferon gamma therapy for alveolar echinococcosis. Clin Infect Dis 26:492-494

Knapp S, Branger J, van der Poll T (2002) Advances in research of the inflammatory response: the importance of Toll-like receptors. Wien Med Wochenschr 152:552-554

Liance M, Ricard-Blum S, Emery I, Houin R, Vuitton DA (1998) Echinococcus multilocularis infection in mice: in vivo treatment with a low dose of IFN-gamma decreases metacestode growth and liver fibrogenesis. Parasite 5:231-237

Lightowlers MW, Rickard MD (1988) Excretory-secretory products of helminth parasites: effects on host immune responses. Parasitology 96(Suppl):S123-S166

Lutz MB, Kukutsch N, Ogilvie AL, Rossner S, Koch F, Romani N, Schuler G (1999) An advanced culture method for generating large quantities of highly pure dendritic cells from mouse bone marrow. J Immunol Methods 223:77-92

Mejri N, Gottstein B (2009) Echinococcus multilocularis metacestode metabolites contain a cysteine protease that digests eotaxin, a CC pro-inflammatory chemokine. Parasitol Res 105:1253-1260

Meijri N, Hemphill A, Gottstein B (2010) Triggering and modulation of the host-parasite interplay by Echinococcus multilocularis: a review. Parasitology 137:557-568

Osada Y, Ohtani T, Une T, Ogawa H, Nomoto K (1982) Enhancement of non-specific resistance to Pseudomonas pneumonia by a synthetic derivative of muramoyl dipeptide in immunosuppressed guinea pigs. J Gen Microbiol 128:2361-2370

Pastrana DV, Raghavan N, FitzGerald P, Eisinger SW, Metz C, Bucala R, Schleimer RP, Bickel C, Scott AL (1998) Filarial nematode parasites secrete a homologue of the human cytokine macrophage migration inhibitory factor. Infect Immun 66:5955-5963

Reuben JM, Tanner CE (1983) Protection against experimental echinococcosis by non-specifically stimulated peritoneal cells. Parasite Immunol 5:61-66

Reuben JM, Tanner CE, Rau ME (1978) Immunoprophylaxis with BCG of experimental Echinococcus multilocularis infections. Infect Immun 21:135-139

Reuter S, Merkle M, Brehm K, Kern P, Manfras B (2003) Effect of amphotericin B on larval growth of Echinococcus multilocularis. Antimicrob Agents Chemother 47:620-625

Rock FL, Hardiman G, Timans JC, Kastelein RA, Bazan JF (1998) A family of human receptors structurally related to Drosophila Toll. Proc Natl Acad Sci USA 95:588-593

Schijns VE (2000) Immunological concepts of vaccine adjuvant activity. Curr Opin Immunol 12:456-463 
Siles-Lucas M, Felleisen RS, Hemphill A, Wilson W, Gottstein B (1998) Stage-specific expression of the 14-3-3 gene in Echinococcus multilocularis. Mol Biochem Parasitol 91:281-293

Siles-Lucas M, Merli M, Mackenstedt U, Gottstein B (2003) The Echinococcus multilocularis 14-3-3 protein protects mice against primary but not secondary alveolar echinococcosis. Vaccine 21:431-439

Thoma-Uszynski S, Stenger S, Takeuchi O, Ochoa MT, Engele M, Sieling PA, Barnes PF, Rollinghoff M, Bolcskei PL, Wagner M, Akira S, Norgard MV, Belisle JT, Godowsk PJ, Bloom BR, Modlin RL (2001) Induction of direct antimicrobial activity through mammalian Toll-like receptors. Science 291:1544-1547

Vuitton DA (2003) The ambiguous role of immunity in echinococcosis: protection of the host or of the parasite? Acta Trop 85:119-132
Vuitton DA, Gottstein B (2010) Echinococcus multilocularis and its intermediate host: a model of parasite-host interplay. J Biomed Biotechnol 2010:923193, Epub 2010 Mar 21

Vuitton DA, Bresson-Hadni S, Laroche L, Kaiserlian D, GuerretStocker S, Bresson JL, Gillet M (1989) Cellular immune response in Echinococcus multilocularis infection in humans. II. Natural killer cell activity and cell subpopulations in the blood and in the periparasitic granuloma of patients with alveolar echinococcosis. Clin Exp Immunol 78:67-74

Walker M, Baz A, Dematteis S, Stettler M, Gottstein B, Schaller J, Hemphill A (2004) Isolation and characterization of a secretory component of Echinococcus multilocularis metacestodes potentially involved in modulating the host-parasite interface. Infect Immun 72:527-536 